\title{
Determination of Absolute Configuration and Biological Activity of New Immunosuppressants, Mycestericins D, E, F and $\mathbf{G}^{\dagger}$
}

\author{
Tetsuro Fujita $^{*+\dagger}$, Norimitsu Hamamichi, Masatoshi Kiuchi ${ }^{\dagger \dagger}$, Tohru Matsuzaki, \\ Yuki Kitao, Kenichiro InOue ${ }^{\dagger \dagger}$, Ryoji Hirose ${ }^{\text {a }}$, Masahiko Yoneta ${ }^{a}$, \\ Shigeo SAsaki ${ }^{\mathrm{a}}$ and Kenji ChIBA ${ }^{\mathrm{b}}$ \\ Faculty of Pharmaceutical Sciences, Kyoto University, \\ Sakyo-ku, Kyoto 606-01, Japan \\ ${ }^{a}$ Research Laboratory, Taito Company, Ltd., \\ 1-26 Higashi Shiriike-Shinmachi, Nagata-ku, Kobe 653, Japan \\ ${ }^{\mathrm{b}}$ Research Laboratories, Yoshitomi Pharmaceutical Industries, Ltd., \\ 3-7-25 Koyata, Iruma-shi, Saitama 358, Japan
}

(Received for publication January 10, 1996)

\begin{abstract}
Mycestericins D, E, F and $\mathrm{G}$ were isolated from the culture broth of Mycelia sterilia ATCC 20349 as potent immunosuppressants. Mycestericins $F$ and $G$ were identical with dihydromycestericins $\mathrm{D}$ and $\mathrm{E}$, respectively. Their absolute configurations were determined by use of the modified MosHER's method and by comparison of the CD spectra of their benzoate derivatives with those of synthetic analogs. Mycestericins D, E, F and G suppressed the proliferation of lymphocytes in the mouse allogeneic mixed lymphocyte reaction.
\end{abstract}

We have isolated a potent immunosuppressant, ISP-I ${ }^{2)}$ (1: myriocin $^{3,4)}$, thermozymocidin ${ }^{57}$ ), from the culture broth of Isaria sinclairii (ATCC 24400) and reported that it was 10 to 100 times more potent than cyclosporin $\mathrm{A}$ in terms of suppressing lymphocyte proliferation in mouse allogeneic mixed lymphocyte reaction (MLR) in vitro, and allo-reactive cytotoxic $\mathrm{T}$ lymphocytes generation and $\mathrm{T}$ cell-dependent antibody production in vivo. A search for new compounds having more potent activity than $\mathbf{1}$ led to the isolation of mycestericins A, B,

Fig. 1. The structures of ISP-I and mycestericins D, E, F, G

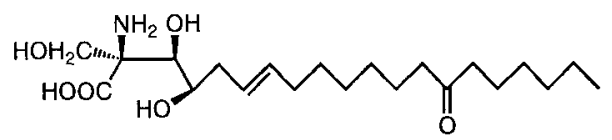

ISP-I (1: Myriocin, Thermozymocidin)
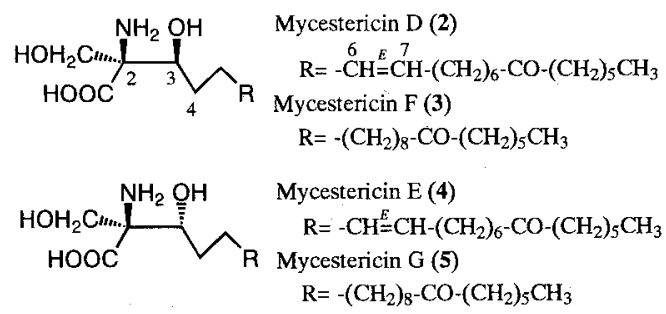

C, D (2) and E (4) as minor analogs from an ISP-Iproducing strain, Mycelia sterilia (ATCC 20349) ${ }^{6}$. The plane structures of $\mathbf{2}$ and $\mathbf{4}$ were clarified and it was established that they are in a diastereo isomeric relationship to each other ${ }^{6}$. However, their absolute configurations were not elucidated. Further study led to the isolation of two more new active compounds, mycestericins $F(3)$ and $G(5)$, from the same strain.

The present paper describes in detail the elucidation of the structures and absolute configurations ${ }^{7}$, as well as the biological activities, of $2,3,4$ and 5 .

\section{Results and Discussion}

Isolation of Mycestericins D, E, F and G

Mycestericins D (2) and E (4) were isolated from the culture broth of $M$. sterilia as described in a previous paper ${ }^{6}$. Further examination of the crude fraction containing 2 and 4 by means of preparative HPLC gave 3 and 5.

\footnotetext{
Determination of Plane Structures of Mycestericins F and G

Mycestericin F

Mycestericin F (3) was obtained as a white powder.
}

\footnotetext{
† Fungal Metabolites. Part 22. (see ref. 1)

Present address:

${ }^{\dagger}$ Faculty of Pharmaceutical Sciences, Setsunan University, 45-1 Nagaotoge, Hirakata, Osaka 573-01, Japan

f†† Research Laboratories, Yoshitomi Pharmaceutical Industries, Ltd., 3-7-25 Koyata, Iruma-shi, Saitama 358, Japan.

+t†† Dept. of Pharmacognosy, Gifu Pharmaceutical University, 5-6-1 Mitahora-Higashi, Gifu 502, Japan.
} 
The molecular formula was determined as $\mathrm{C}_{21} \mathrm{H}_{41} \mathrm{NO}_{5}$ by HRFAB-MS (Calcd for $\mathrm{C}_{21} \mathrm{H}_{42} \mathrm{NO}_{5}: 388.3065$, Found: 388.3049). The formula has two hydrogen atoms more than that of 2 . The IR and ${ }^{1} \mathrm{H}$ NMR spectra of 3 were similar to those of $\mathbf{2}$. However, the C-6, 7 olefin signals of 2 (IR: $970 \mathrm{~cm}^{-1},{ }^{1} \mathrm{H}$ NMR: $\delta 5.4 \sim 5.5$, $-\mathrm{CH}=\mathrm{CH}-$ and $\delta 2.0,-\mathrm{CH}_{2}-\mathrm{CH}=\mathrm{CH}-\mathrm{CH}_{2}-$ ) were absent in the spectra of 3 . Instead, a signal due to $-\mathrm{CH}_{2}-\times 4$ at $1.29 \mathrm{ppm}$ was observed in the ${ }^{1} \mathrm{H}$ NMR spectrum of 3 . These data suggested that 3 is the 6,7 dihydro derivative of $\mathbf{2}$. It was confirmed by the catalytic hydrogenation of $\mathbf{2}$.

\section{Mycestericin G}

Mycestericin $\mathrm{G}$ (5), $\mathrm{C}_{21} \mathrm{H}_{41} \mathrm{NO}_{5}$ (HRFAB-MS Calcd for $\mathrm{C}_{21} \mathrm{H}_{42} \mathrm{NO}_{5}:$ 388.3065, Found: 388.3032), was obtained as a white powder. Although the ${ }^{1} \mathrm{H}$ NMR spectrum of $\mathbf{5}$ was very similar to that of $\mathbf{3}$, the signal of methylene protons $(\delta: 1.53,2 \mathrm{H})$ at $\mathrm{C}-4$ of 3 was replaced by signals at $\delta 1.62$ and $\delta 1.36$ in the spectrum of 5 . The spectra (IR, ${ }^{1} \mathrm{H}$ NMR, MS) of 5 were identical with those of dihydromycestericin $\mathrm{E}$.

\section{Absolute Structures of Mycestericins $D, E, F$ and $G$}

The absolute configurations of $\mathbf{2}, \mathbf{3}, \mathbf{4}$ and $\mathbf{5}$ were determined by comparison of the $\mathrm{CD}$ spectra of their $N, O, O^{\prime}$-tribenzoyl methyl esters $(\mathbf{2 2} \sim \mathbf{2 5})$ with those of synthetic compounds 12, 13, 20 and 21 . The syntheses are outlined in Scheme 1. Stereoselective acylation of the protected D-serine $6^{8,9)}$ with stearoyl chloride afforded the $\beta$-keto ester 7. The ketonic carbonyl oxygen and the ester carbonyl carbon were confirmed to be oriented to the same side in the same plane, based on NOE experiments (Fig. 2). Therefore, it is considered that the stereo-

Scheme 1. Syntheses of 12, 13, 20 and 21.
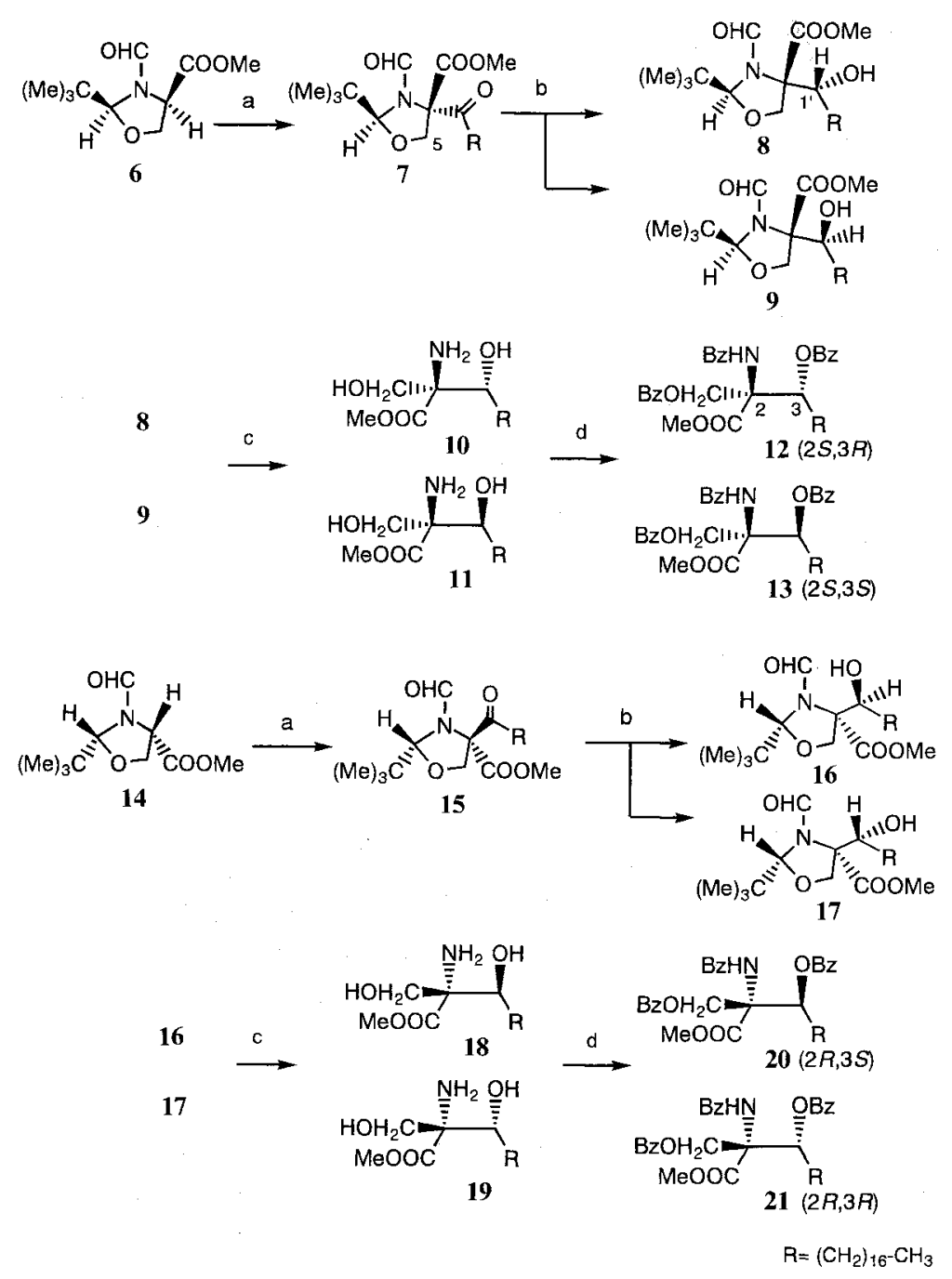

Reagents: a) $\mathrm{CH}_{3}\left(\mathrm{CH}_{2}\right)_{16} \mathrm{COCl}$ (1.2 eq.), LDA (1.2 eq.), THF, $-100^{\circ} \mathrm{C}$; b) $\mathrm{NaBH}_{4}$ (1.2 eq.), $\mathrm{MeOH}$; c) $6 \mathrm{~N} \mathrm{HCl}, \mathrm{MeOH}, 80^{\circ} \mathrm{C}, 1$ hour; d) $\mathrm{Bz}_{2} \mathrm{O}, \mathrm{Et}_{3} \mathrm{~N}, 40^{\circ} \mathrm{C}, 2$ hours. 
Fig. 2. ${ }^{1} \mathrm{H}-{ }^{1} \mathrm{H}$ correlations of 7 obtained in an NOE experiment
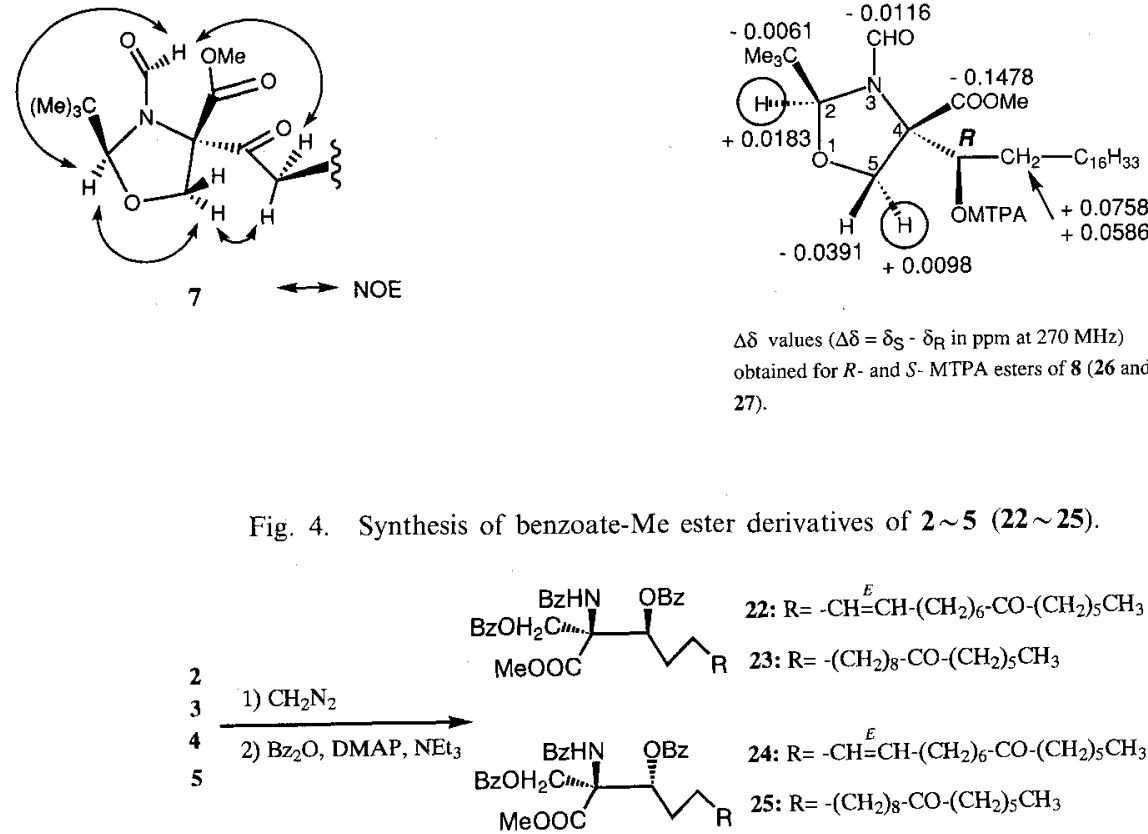

Fig. 5. CD spectra of 13, 21, 22 and 23 (in $\mathrm{MeOH}$ ).

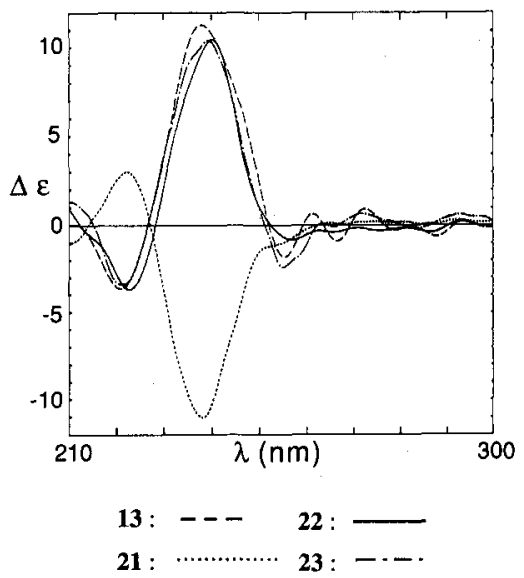

selective reduction of the $\beta$-keto ester 7 with $\mathrm{NaBH}_{4}$ gave the $\left(1^{\prime} R\right)$-hydroxy compound $\mathbf{8}$ as the major product and the $\left(1^{\prime} S\right)$-hydroxy compound 9 as the minor product by hydride attack from the less hindered side because of the presence of the formyl group at the re-face of the ketone. The absolute configuration of the ( $\left(1^{\prime} R\right)$-hydroxy group of the major product 8 was confirmed by means of the modified Mosher's method ${ }^{10)}$ (Fig. 3). Consequently, the 1'-hydroxy group of the minor product 9 should have $S$-configuration. Treatment of 8 and 9 with $6 \mathrm{~N} \mathrm{HCl}$ afforded the esters 10 and 11, which were benzoylated to give $\mathbf{1 2}$ and $\mathbf{1 3}$, respectively. By a similar
Fig. 3. Application of the modified MOSHER's method to 8 .

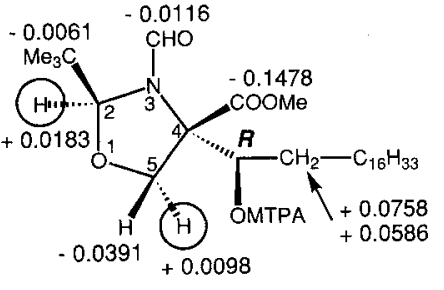

$\Delta \delta$ values $\left(\Delta \delta=\delta_{\mathrm{S}}-\delta_{\mathrm{R}}\right.$ in ppm at $\left.270 \mathrm{MHz}\right)$ obtained for $R$ - and $S$ - MTPA esters of 8 (26 and 27).

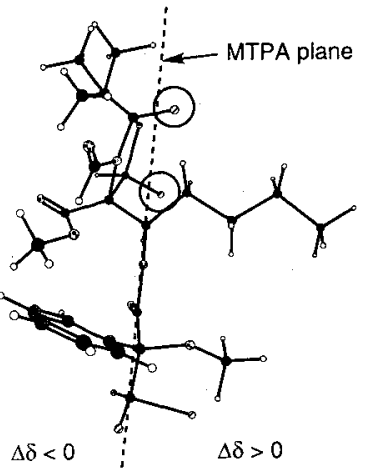

Fig. 6. CD spectra of 12, 20, 24 and 25 (in $\mathrm{MeOH}$ ).

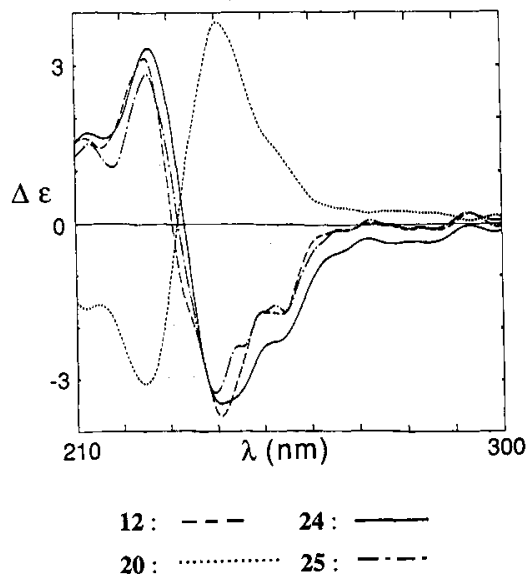

procedure, $20(2 R, 3 S)$ and $21(2 R, 3 R)$ as major and minor products, respectively, were prepared from protected $\mathrm{L}$-serine $\mathbf{1 4}^{8)}$. On the other hand, the natural products $2 \sim 5$ were treated with $\mathrm{CH}_{2} \mathrm{~N}_{2}$, followed by benzoylation with benzoic anhydride $\left(\mathrm{Bz}_{2} \mathrm{O}\right)$ and $N, N$ dimethylaminopyridine (DMAP) in $\mathrm{Et}_{3} \mathrm{~N}$ to afford the $N, O, O^{\prime}$-tribenzoyl methyl esters $(22 \sim 25$, Fig. 4). As shown in Fig. 5, the CD curves of $\mathbf{2 2}$ and $\mathbf{2 3}$ showed good coincidence with that of $\mathbf{1 3}(2 S, 3 S)$. Further, the CD curves of $\mathbf{2 4}$ and $\mathbf{2 5}$ showed good coincidence with that of $12(2 S, 3 R)$ (Fig. 6). The 3-hydroxy group of 4 was also determined to have $R$-configuration by means 
Fig. 7. Application of MosHER's method to mycestericin E.

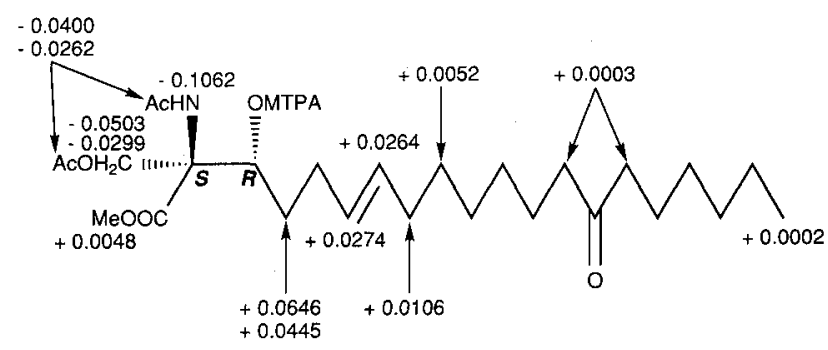

$\Delta \delta$ values $\left(\Delta \delta=\delta_{\mathrm{S}}-\delta_{\mathrm{R}}\right.$ in ppm at $600 \mathrm{MHz}$ ) obtained for $R$ - and $S$ - MTPA esters of diacetylmycestericin $E$ methyl ester (28 and 29).

Fig. 8. The, structures of ISP-I-55 and FTY720.

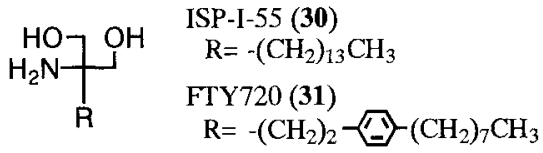

of the modified MosHER's method (Fig. 7).

Consequently, the absolute configurations of mycestericins $\mathrm{D}, \mathrm{E}, \mathrm{F}$ and $\mathrm{G}$ were determined to be $2(2 S, 3 S)$, $3(2 S, 3 S), 4(2 S, 3 R)$ and $5(2 S, 3 R)$, as shown in Fig. 1.

The structures of $\mathbf{4}$ and $\mathbf{5}$ were unambiguously confirmed by total synthesis ${ }^{7)}$.

\section{Immunosuppressive Activity}

The effect of 2, 3, 4 and 5 on the mouse allogeneic mixed lymphocyte reaction $(\mathrm{MLR})^{2)}$ was examined in comparison with that of 1 . Table 1 shows the $\mathrm{IC}_{50}$ values of $1 \sim 5$. From these results, the following structureactivity relationships were apparent. 1) The hydroxy group at $\mathrm{C}-4$ has no effect on the activity $(\mathbf{1} \rightarrow \mathbf{2})$. 2) The configuration of the 3-hydroxyl group is unimportant $(\mathbf{2} \rightarrow \mathbf{4}, \mathbf{3} \rightarrow \mathbf{5})$. 3) The C-6 olefin group increases the activity $(\mathbf{2} \rightarrow \mathbf{3}, \mathbf{4} \rightarrow \mathbf{5})$. These structure-activity relationships provided clues that led to the development of 2-substituted 2-amino-1,3-propanediols, ISP-I-55 $(\mathbf{3 0})^{11)}$ and FTY720 (31) ${ }^{12)}$ (Fig. 8).

\section{Experimental}

\section{General Methods}

Melting points were determined on a Yanagimoto micro melting point apparatus without correction. Optical rotations were measured with a Jasco DIP-181 or Jasco DIP-140 digital polarimeter. UV and IR spectra were taken on a Shimadzu UV-2200 UV-VIS recording spectrophotometer and a Shimadzu IR-435 infrared spectrophotometer, respectively. CD spectra were measured on a Jasco J-720 spectrophotometer. The concentration of benzoate derivatives was determined
Table 1. Effect of ISP-I and mycestericins on mouse allogeneic MLR.

\begin{tabular}{|c|c|}
\hline Compound & $\mathrm{IC}_{50}(\mathrm{nM})$ \\
\hline ISP-I (1) & 8.0 \\
\hline Mycestericin D (2) & 16 \\
\hline E (4) & 13 \\
\hline $\mathbf{F}(\mathbf{3})$ & 120 \\
\hline$G(5)$ & 370 \\
\hline
\end{tabular}

from the UV spectra by using a calibration curve for propyl benzoate. ${ }^{1} \mathrm{H}$ NMR and ${ }^{13} \mathrm{C}$ NMR spectra were taken on a JEOL FX-200, JEOL EX-270, Bruker AC300 or Bruker AM-600 spectrometer with TMS as an internal standard. Mass spectra (EI-MS and FAB-MS) were taken on a JEOL JMS-01SG, JEOL JMS-HX100 or JMS-HX110 spectrometer. HPLC was performed on a Shimadzu LC-8A system [eluent $\mathrm{MeOH}-\mathrm{H}_{2} \mathrm{O}$ $(65: 35 \mathrm{~V} / \mathrm{v})$; column temperature $40^{\circ} \mathrm{C}$; UV detector $(210 \mathrm{~nm})]$. Analytical HPLC was carried out with a YMC-ODS AM 313 column (i.d. $6 \mathrm{~mm} \times 250 \mathrm{~mm}$ ) (flow rate $1.0 \mathrm{ml} / \mathrm{minute}$ ). Preparative $\mathrm{HPLC}$ was carried out with a YMC-ODS SH-343-5 column (i.d. $20 \mathrm{~mm} \times 250$ $\mathrm{mm}$ ) (flow rate $7.0 \mathrm{ml} /$ minute). Analytical TLC and preparative TLC were performed on Kieselgel 60 F254 (Merck) and Kieselgel 60 PF254 (Merck), respectively. Organic extracts of reaction mixtures were washed successively with $1 \mathrm{~N} \mathrm{HCl,} 5 \% \mathrm{NaHCO}_{3}$ and saturated aqueous $\mathrm{NaCl}$ and dried over anhydrous magnesium sulfate, unless otherwise specified.

Isolation and Purification

Mycestericins D (2) and E (4) were isolated from the culture broth of $M$. sterilia as described in the previous paper $^{6)}$. A crude fraction of 2 and $4(650 \mathrm{mg})$ containing 3 and 5 was subjected to preparative HPLC to give $\mathbf{3}$ (5.8 $\mathrm{mg}$; retention time, $109 \sim 112$ minutes) and 5 ( $9.0 \mathrm{mg}$; retention time, $115 \sim 118$ minutes).

3: MP $190 \sim 191.5^{\circ} \mathrm{C}$. $[\alpha]_{\mathrm{D}}^{25}-7.94^{\circ}(c 0.106, \mathrm{MeOH})$. IR $v_{\max }(\mathrm{KBr}) \mathrm{cm}^{-1}: 3375,3100,2910,2830,1705,1665$, 1580, 1045. ${ }^{1} \mathrm{H}$ NMR (CD 3 OD, $\left.300 \mathrm{MHz}\right) \delta: 3.98(1 \mathrm{H}$, d, $J=11.1 \mathrm{~Hz}, 21 \mathrm{H}), 3.82(1 \mathrm{H}, \mathrm{d}, J=11.1 \mathrm{~Hz}, 21-\mathrm{H})$, $3.81(1 \mathrm{H}, \mathrm{m}, 3-\mathrm{H}), 2.43\left(4 \mathrm{H}, \mathrm{t}, J=7.3 \mathrm{~Hz}, 13-, 15-\mathrm{H}_{2}\right)$, $1.53\left(6 \mathrm{H}, \mathrm{m}, 4-, 12-, 16-\mathrm{H}_{2}\right), 1.29(20 \mathrm{H}, \mathrm{m}, 5-\sim 11-$, $\left.17-\sim 19-\mathrm{H}_{2}\right), 0.90\left(3 \mathrm{H}, \mathrm{t}, J=6.3 \mathrm{~Hz}, 20-\mathrm{H}_{3}\right)$. FAB-MS $m / z 388\left[(\mathrm{M}+\mathbf{H})^{+}\right], 282,246$.

5: MP $187 \sim 189^{\circ} \mathrm{C}$. $[\alpha]_{\mathrm{D}}^{25}-6.81^{\circ}(c 0.106, \mathrm{MeOH})$. IR $v_{\max }(\mathrm{KBr}) \mathrm{cm}^{-1}: 3400,3100,2910,2830,1710,1660$, $1580,1045 .{ }^{1} \mathrm{H}$ NMR $\left(\mathrm{CD}_{3} \mathrm{OD}, 300 \mathrm{MHz}\right) \delta: 3.93(1 \mathrm{H}$, $\mathrm{d}, J=11.1 \mathrm{~Hz}, 21 \mathrm{H}), 3.83(1 \mathrm{H}, \mathrm{m}, 3-\mathrm{H}), 3.81(1 \mathrm{H}, \mathrm{d}$, $J=11.1 \mathrm{~Hz}, 21-\mathrm{H}), 2.44\left(4 \mathrm{H}, \mathrm{t}, J=7.13 \mathrm{~Hz}, 13-, 15-\mathrm{H}_{2}\right)$, $1.62(1 \mathrm{H}, \mathrm{m}, 4-\mathrm{H}), 1.54$ (4H, qui, $J=6.5 \mathrm{~Hz}, 12-, 16-\mathrm{H}_{2}$ ), $1.36(1 \mathrm{H}, \mathrm{m}, 4-\mathrm{H}), 1.30\left(20 \mathrm{H}, \mathrm{m}, 5-\sim 11-, 17-\sim 19-\mathrm{H}_{2}\right)$, $0.90\left(3 \mathrm{H}, \mathrm{t}, J=6.6 \mathrm{~Hz}, 20-\mathrm{H}_{3}\right)$. FAB-MS $m / z 388$ $\left[(\mathrm{M}+\mathrm{H})^{+}\right], 282,246$. 
Mouse Allogeneic Mixed Lymphocyte Reaction
(MLR)

The effect of mycestericins on mouse allogeneic MLR was examined by the method described in our previous paper $^{2)}$.

\section{Hydrogenation of 2}

A solution of $2(10.0 \mathrm{mg})$ in $\mathrm{MeOH}(30 \mathrm{ml})$ was subjected to hydrogenation over $5 \%$ palladium carbon $(20 \mathrm{mg})$. The catalyst was filtered off and the solvent was evaporated. The residue was purified by preparative HPLC [eluent $\mathrm{MeOH}-\mathrm{H}_{2} \mathrm{O}(70: 30 \mathrm{v} / \mathrm{v})$; flow rate $8.0 \mathrm{ml} /$ minute] to give dihydromycestericin $\mathrm{D}$ as a white powder $(9.39 \mathrm{mg}, 93.4 \%)$. This compound was identical with 3.

\section{Hydrogenation of $\mathbf{4}$}

By a procedure similar to that used for the preparation of $\mathbf{3}$ from $2,5(9.65 \mathrm{mg})$ was obtained as a white powder in $96.0 \%$ yield from $\mathbf{4}(10.0 \mathrm{mg})$.

Methyl (2S,4S)-2-t-Butyl-3-formyl-4-octadecanoyloxazolidine-4-carboxylate (7)

Compound 7 was synthesized from methyl $(2 S, 4 R)$-2$t$-butyl-3-formyloxazolidine-4-carboxylate $(6,1.27 \mathrm{~g})$ and stearoyl chloride $(2.14 \mathrm{~g})$ in the reported manner. ${ }^{9)}$ The product was purified to give 7 as an oil $(1.302 \mathrm{~g}, 45.8 \%)$ by chromatography on a silica gel column using $n$ hexane-EtOAc $(9: 1) .[\alpha]_{\mathrm{D}}^{25}+3.01^{\circ}\left(c 1.22, \mathrm{CHCl}_{3}\right)$. IR $v_{\max }\left(\mathrm{CHCl}_{3}\right) \mathrm{cm}^{-1}: 3000,2915,2850,1750,1725$, $1680,1280,1230 .{ }^{1} \mathrm{H}$ NMR $\left(\mathrm{CDCl}_{3}, 270 \mathrm{MHz}\right) \delta: 8.32$, $8.25(1 \mathrm{H}, 2 \mathrm{~s}, \mathrm{CHO}), 5.48,5.08(1 \mathrm{H}, 2 \mathrm{~s}, 2-\mathrm{H}), 4.71,4.61$ $(1 \mathrm{H}, 2 \mathrm{~d}, J=10.2$ and $9.0 \mathrm{~Hz}, 5-\mathrm{H}), 4.16,4.08(1 \mathrm{H}, 2 \mathrm{~d}$, $J=10.2$ and $9.0 \mathrm{~Hz}, 5-\mathrm{H}), 3.88,3.85\left(3 \mathrm{H}, 2 \mathrm{~s}, \mathrm{OCH}_{3}\right)$, $2.68\left(1 \mathrm{H}, \mathrm{dt}, J=17.8\right.$ and $\left.7.6 \mathrm{~Hz}, 2^{\prime}-\mathrm{H}\right), 2.49(1 \mathrm{H}, \mathrm{m}$, $\left.2^{\prime}-\mathrm{H}\right), 1.60\left(2 \mathrm{H}, \mathrm{m}, 3^{\prime}-\mathrm{H}_{2}\right), 1.25\left(28 \mathrm{H}, \mathrm{m}, 4^{\prime}-\sim 17^{\prime}-\mathrm{H}_{2}\right)$, $0.98,0.94(9 \mathrm{H}, 2 \mathrm{~s}, t-\mathrm{Bu}), 0.88\left(3 \mathrm{H}, \mathrm{t}, J=6.5 \mathrm{~Hz}, 18^{\prime}-\mathrm{H}_{3}\right)$. Anal. Calcd for $\mathrm{C}_{28} \mathrm{H}_{51} \mathrm{NO}_{5}: \mathrm{C} 69.82, \mathrm{H} 10.67, \mathrm{~N} 2.91$. Found: C 69.74, H 10.91, N 2.81.

Methyl $(2 R, 4 R)$-2- $t$-Butyl-3-formyl-4-octadecanoyloxazolidine-4-carboxylate (15)

By a procedure similar to that used for the preparation of $7,15(1.515 \mathrm{~g})$ was obtained as an oil in $53.3 \%$ yield from methyl $(2 R, 4 S)$-2- $t$-butyl-3-formyloxazolidine-4carboxylate $(14,1.27 \mathrm{~g})$ and stearoyl chloride $(2.14 \mathrm{~g})$. $[\alpha]_{\mathrm{D}}^{25}-2.20^{\circ}$ (c 2.11, $\left.\mathrm{CHCl}_{3}\right)$. IR $v_{\max }\left(\mathrm{CHCl}_{3}\right) \mathrm{cm}^{-1}$ : $3000,2915,2850,1750,1725,1680,1280,1230 .{ }^{1} \mathrm{H}$ NMR $\left(\mathrm{CDCl}_{3}, 270 \mathrm{MHz}\right) \delta: 8.32,8.25(1 \mathrm{H}, 2 \mathrm{~s}, \mathrm{CHO}), 5.48$, $5.08(1 \mathrm{H}, 2 \mathrm{~s}, 2-\mathrm{H}), 4.72,4.61(1 \mathrm{H}, 2 \mathrm{~d}, J=10.2$ and $9.0 \mathrm{~Hz}, 5-\mathrm{H}), 4.16,4.08(1 \mathrm{H}, 2 \mathrm{~d}, J=10.2$ and $9.0 \mathrm{~Hz}$, $5-\mathrm{H}), 3.88,3.85\left(3 \mathrm{H}, 2 \mathrm{~s}, \mathrm{OCH}_{3}\right), 2.68(1 \mathrm{H}, \mathrm{dt}, J=17.8$ and $\left.7.6 \mathrm{~Hz} 2^{\prime}-\mathrm{H}\right), 2.49\left(1 \mathrm{H}, \mathrm{m}, 2^{\prime}-\mathrm{H}\right), 1.61\left(2 \mathrm{H}, \mathrm{m}, 3^{\prime}-\mathrm{H}_{2}\right)$, $1.25\left(28 \mathrm{H}, \mathrm{m}, 4^{\prime}-\sim 17^{\prime}-\mathrm{H}_{2}\right), 0.98,0.94(9 \mathrm{H}, 2 \mathrm{~s}, t-\mathrm{Bu}), 0.88$ $\left(3 \mathrm{H}, \mathrm{t}, J=6.6 \mathrm{~Hz}, 18^{\prime}-\mathrm{H}_{3}\right)$. Anal. Calcd for $\mathrm{C}_{28} \mathrm{H}_{51} \mathrm{NO}_{5}$ : C 69.82, H 10.67, N 2.91. Found: C 69.94, H 10.93, N 3.01 .
Methyl $(2 S, 4 S)$-2-t-Butyl-3-formyl-4-(1(R)-hydroxyoctadecyl)oxazolidine-4-carboxylate (8) and Methyl $\overline{(2 S, 4 S)}$-2-t-Butyl-3-formyl-4-(1(S)-hydroxyoctadecyl)oxazolidine-4-carboxylate (9)

Sodium borohydride $(10 \mathrm{mg})$ was added to a solution of $7(100 \mathrm{mg})$ in $\mathrm{MeOH}(15 \mathrm{ml})$ at $0^{\circ} \mathrm{C}$. After 15 minutes, $\mathrm{H}_{2} \mathrm{O}(30 \mathrm{ml})$ was added to the mixture, then $\mathrm{MeOH}$ was evaporated off. The $\mathrm{H}_{2} \mathrm{O}$ solution was extracted with $\mathrm{CHCl}_{3}$. The $\mathrm{CHCl}_{3}$ layer was washed with saturated aqueous $\mathrm{NaCl}$, dried and concentrated to give an oily residue. The residue was purified by preparative HPLC [column: YMC-ODS SH-343-5 (i.d. $20 \mathrm{~mm} \times 250 \mathrm{~mm}$ ), mobile phase: $\mathrm{MeOH}-\mathrm{H}_{2} \mathrm{O}(93: 7)$, flow rate: $8.0 \mathrm{ml} /$ minute, temperature: $40^{\circ} \mathrm{C}$, detection: $U V$ at $210 \mathrm{~nm}$ ] to give $8(69.8 \mathrm{mg}, 69.5 \%)$ and $9(14.3 \mathrm{mg}, 14.2 \%)$.

8: $[\alpha]_{\mathrm{D}}^{25}+28.45^{\circ}(c 0.113, \mathrm{MeOH}) . \mathrm{IR} v_{\max }\left(\mathrm{CHCl}_{3}\right)$ $\mathrm{cm}^{-1}: 3350,3000,2905,2840,1755,1730,1665,1215$. ${ }^{1} \mathrm{H}$ NMR $\left(\mathrm{CDCl}_{3}, 270 \mathrm{MHz}\right) \delta: 8.70,8.46(1 \mathrm{H}, 2 \mathrm{~s}$, CHO), 5.39, $4.99(1 \mathrm{H}, 2 \mathrm{~s}, 2-\mathrm{H}), 5.03(1 \mathrm{H}, \mathrm{d}, J=11.2 \mathrm{~Hz}$, $\mathrm{OH}), 4.50,4.40(1 \mathrm{H}, 2 \mathrm{~d}, J=9.4 \mathrm{~Hz}, 5-\mathrm{H}), 3.95(1 \mathrm{H}$, br t-like, $\left.J=10.7 \mathrm{~Hz}, 1^{\prime}-\mathrm{H}\right), 3.90(1 \mathrm{H}, \mathrm{d}, J=9.4 \mathrm{~Hz}, 5-\mathrm{H})$, $3.83,3.80(3 \mathrm{H}, 2 \mathrm{~s}, \mathrm{OMe}), 1.64\left(1 \mathrm{H}, \mathrm{m}, 2^{\prime}-\mathrm{H}\right), 1.46(1 \mathrm{H}$, $\left.\mathrm{m}, 2^{\prime}-\mathrm{H}\right), 1.25\left(30 \mathrm{H}\right.$, br s, $\left.3^{\prime}-\sim 17^{\prime}-\mathrm{H}_{2}\right), 1.00,0.94(9 \mathrm{H}$, $2 \mathrm{~s},-\mathrm{Bu}), 0.88\left(3 \mathrm{H}, \mathrm{t}, J=6.3 \mathrm{~Hz}, 18^{\prime}-\mathrm{H}_{3}\right)$. Anal. Calcd for $\mathrm{C}_{28} \mathrm{H}_{53} \mathrm{NO}_{5}: \mathrm{C} 69.52, \mathrm{H} 11.04, \mathrm{~N} 2.90$. Found: C 69.72, H 11.25, N 2.94 .

9: $[\alpha]_{\mathrm{D}}^{25}+1.13^{\circ}(c 0.053, \mathrm{MeOH}) . \mathrm{IR} v_{\max }\left(\mathrm{CHCl}_{3}\right)$ $\mathrm{cm}^{-1}: 3370,3000,2905,2840,1750$ (sh), 1725, 1660, 1215. ${ }^{1} \mathrm{H}$ NMR $\left(\mathrm{CDCl}_{3}, 270 \mathrm{MHz}\right) \delta: 8.39,8.19(1 \mathrm{H}, 2 \mathrm{~s}$, $\mathrm{CHO}), 5.33,5.16(1 \mathrm{H}, \mathrm{m}$ and $\mathrm{br}, \mathrm{OH}), 4.85(1 \mathrm{H}, \mathrm{s}$, $2-\mathrm{H}), 4.36,4.01(1 \mathrm{H}, 2 \mathrm{~d}, J=8.9$ and $9.2 \mathrm{~Hz}, 5-\mathrm{H}), 4.17$ $\left(1 \mathrm{H}, \mathrm{m}, 1^{\prime}-\mathrm{H}\right), 3.92,3.66(1 \mathrm{H}, 2 \mathrm{~d}, J=8.9$ and $9.2 \mathrm{~Hz}$, $5-\mathrm{H}), 3.79,3.78(3 \mathrm{H}, 2 \mathrm{~s}, \mathrm{OMe}), 1.79\left(1 \mathrm{H}, \mathrm{m}, 2^{\prime}-\mathrm{H}\right), 1.59$ $\left(1 \mathrm{H}, \mathrm{m}, 2^{\prime}-\mathrm{H}\right), 1.25\left(30 \mathrm{H}\right.$, br s, $\left.3^{\prime}-\sim 17^{\prime}-\mathrm{H}_{2}\right), 1.01,0.97$ $(9 \mathrm{H}, 2 \mathrm{~s}, t-\mathrm{Bu}), 0.88\left(3 \mathrm{H}, \mathrm{t}, J=6.6 \mathrm{~Hz}, 18^{\prime}-\mathrm{H}_{3}\right)$. Anal. Calcd for $\mathrm{C}_{28} \mathrm{H}_{53} \mathrm{NO}_{5}$ : N 2.90. Found: N 3.02.

Methyl $(2 R, 4 R)$-2-t-Butyl-3-formyl-4-(1(S)-hydroxyoctadecyl)oxazolidine-4-carboxylate (16) and Methyl $(2 R, 4 R)-2-t$-Butyl-3-formyl-4-(1(R)-hydroxyoctadecyl)oxazolidine-4-carboxylate (17)

By a procedure similar to that used for the preparation of $\mathbf{8}$ and $\mathbf{9}, \mathbf{1 6}(67.2 \mathrm{mg})$ and $\mathbf{1 7}(12.1 \mathrm{mg})$ were obtained as oils in yields of 66.9 and $12.0 \%$ from $15(100 \mathrm{mg})$, respectively.

16: $[\alpha]_{\mathrm{D}}^{25}-32.2^{\circ}\left(c 0.116, \mathrm{CHCl}_{3}\right) . \mathrm{IR} v_{\max }\left(\mathrm{CHCl}_{3}\right)$ $\mathrm{cm}^{-1}: 3350,3000,2905,2840,1755,1735,1665,1215$. ${ }^{1} \mathrm{H}$ NMR $\left(\mathrm{CDCl}_{3}, 270 \mathrm{MHz}\right) \delta: 8.70,8.46(1 \mathrm{H}, 2 \mathrm{~s}$, CHO), 5.39, $4.99(1 \mathrm{H}, 2 \mathrm{~s}, 2-\mathrm{H}), 5.03(1 \mathrm{H}, \mathrm{d}, J=11.2 \mathrm{~Hz}$, $\mathrm{OH}), 4.50,4.40(1 \mathrm{H}, 2 \mathrm{~d}, J=9.4 \mathrm{~Hz}, 5-\mathrm{H}), 3.95(1 \mathrm{H}, \mathrm{brt}$ like, $\left.J=10.7 \mathrm{~Hz}, 1^{\prime}-\mathrm{H}\right), 3.90(1 \mathrm{H}, \mathrm{d}, J=9.4 \mathrm{~Hz}, 5-\mathrm{H})$, $3.83,3.80(3 \mathrm{H}, 2 \mathrm{~s}, \mathrm{OMe}), 1.64\left(1 \mathrm{H}, \mathrm{m}, 2^{\prime}-\mathrm{H}\right), 1.47(1 \mathrm{H}$, $\left.\mathrm{m}, 2^{\prime}-\mathrm{H}\right), 1.25\left(30 \mathrm{H}\right.$, br s, $\left.3^{\prime}-\sim 17^{\prime}-\mathrm{H}_{2}\right), 0.99,0.94(9 \mathrm{H}$, $2 \mathrm{~s}, t-\mathrm{Bu}), 0.88\left(3 \mathrm{H}, \mathrm{t}, J=6.3 \mathrm{~Hz}, 18^{\prime}-\mathrm{H}_{3}\right)$.

17: $[\alpha]_{\mathrm{D}}^{25}-0.20^{\circ}\left(c\right.$ 0.044, MeOH). IR $v_{\max }\left(\mathrm{CHCl}_{3}\right)$ $\mathrm{cm}^{-1}: 3350,3000,2905,2840,1750$ (sh), 1730, 1660, 1215. ${ }^{1} \mathrm{H} \mathrm{NMR}\left(\mathrm{CDCl}_{3}, 270 \mathrm{MHz}\right) \delta: 8.39,8.19(1 \mathrm{H}, 2 \mathrm{~s}$, $\mathrm{CHO}), 5.33,5.16(1 \mathrm{H}, \mathrm{m}$ and $\mathrm{brs}, \mathrm{OH}), 4.85(1 \mathrm{H}, \mathrm{s}$, 
$2-\mathrm{H}), 4.36,4.01(1 \mathrm{H}, 2 \mathrm{~d}, J=8.9$ and $9.2 \mathrm{~Hz}, 5-\mathrm{H}), 4.18$ $\left(1 \mathrm{H}, \mathrm{m}, \mathrm{l}^{\prime}-\mathrm{H}\right), 3.92,3.66(1 \mathrm{H}, 2 \mathrm{~d}, J=8.9$ and $9.2 \mathrm{~Hz}$, 5-H), 3.79, 3.78 (3H, 2s, OMe), $1.79\left(1 \mathrm{H}, \mathrm{m}, 2^{\prime}-\mathrm{H}\right), 1.58$ $\left(1 \mathrm{H}, \mathrm{m}, 2^{\prime}-\mathrm{H}\right), 1.25\left(30 \mathrm{H}\right.$, br s, 3'- 17'- $\left.\mathrm{H}_{2}\right), 1.01,0.97$ $(9 \mathrm{H}, 2 \mathrm{~s}, t-\mathrm{Bu}), 0.88\left(3 \mathrm{H}, \mathfrak{t}, J=6.6 \mathrm{~Hz}, 18^{\prime}-\mathrm{H}_{3}\right)$.

Methyl 2-Amino-3-hydroxy-2-hydroxymethyleicosanoates $(\mathbf{1 0}(2 S, 3 R), \mathbf{1 1}(2 S, 3 S), \mathbf{1 8}(2 R, 3 S)$ and $19(2 R, 3 R))$

Compound $8(10 \mathrm{mg}), 9(10 \mathrm{mg}), 16(10 \mathrm{mg})$ or 17 $(10 \mathrm{mg})$ was treated with $6 \mathrm{~N}$ methanolic $\mathrm{HCl}$ at $80^{\circ} \mathrm{C}$ for 1 hour. The mixture was applied to an IRA-93zu (eluent, $\mathrm{MeOH}$ ) column and the eluate was concentrated to give a residue. The residue was purified by preparative TLC [solvent, $\left.\mathrm{CHCl}_{3}-\mathrm{MeOH}-\mathrm{H}_{2} \mathrm{O}(65: 35: 2)\right]$ to give $\mathbf{1 0}$ (4.9 mg, 61.2\%), 11 (4.8 mg, 59.9\%), 18 (5.8 mg, 72.4\%) or $19(5.1 \mathrm{mg}, 64.6 \%)$, respectively.

10: $\mathrm{MP} 60 \sim 61^{\circ} \mathrm{C}$. $[\alpha]_{\mathrm{D}}^{25}+25.31^{\circ}\left(c 1.05, \mathrm{CHCl}_{3}\right)$. IR $v_{\text {max }}\left(\mathrm{CHCl}_{3}\right) \mathrm{cm}^{-1}: 3570(\mathrm{sh}), 3450(\mathrm{sh}), 3390,3000$, 2915, 2840, 1730, 1225. ${ }^{1} \mathrm{H}$ NMR (CD $\mathrm{OD}, 270 \mathrm{MHz}$ ) $\delta$ : $3.85(1 \mathrm{H}, \mathrm{d}, J=10.6 \mathrm{~Hz}, 21-\mathrm{H}), 3.74(4 \mathrm{H}, \mathrm{m}, 3-\mathrm{H}$ and $\mathrm{OMe}), 3.51(1 \mathrm{H}, \mathrm{d}, J=10.6 \mathrm{~Hz}, 21-\mathrm{H}), 1.54(1 \mathrm{H}, \mathrm{m}$, 4-H), $1.28\left(31 \mathrm{H}, \mathrm{m}, 4-\mathrm{H}, 5-\sim 19-\mathrm{H}_{2}\right), 0.90(3 \mathrm{H}, \mathrm{t}$, $\left.J=6.3 \mathrm{~Hz}, 20-\mathrm{H}_{3}\right)$. FAB-MS $m / z: 388\left[(\mathrm{M}+\mathrm{H})^{+}\right], 370$, $328,118,55,43$.

11: MP $65 \sim 69.5^{\circ} \mathrm{C} .[\alpha]_{\mathrm{D}}^{25}+3.40^{\circ}\left(c 0.92, \mathrm{CHCl}_{3}\right)$. IR $v_{\max }\left(\mathrm{CHCl}_{3}\right) \mathrm{cm}^{-1}: 3600(\mathrm{sh}), 3500(\mathrm{sh}), 3390,3000$, 2910, 2840, 1730, 1225. ${ }^{1} \mathrm{H}$ NMR (CD 3 OD, 270 MHz) $\delta: 3.88(1 \mathrm{H}, \mathrm{d}, J=10.9 \mathrm{~Hz}, 21-\mathrm{H}), 3.73(3 \mathrm{H}, \mathrm{s}, \mathrm{OMe})$, $3.67(1 \mathrm{H}, \mathrm{dd}, J=10.2$ and $2.0 \mathrm{~Hz}, 3-\mathrm{H}), 3.64(1 \mathrm{H}, \mathrm{d}$, $J=10.9 \mathrm{~Hz}, 21-\mathrm{H}), 1.51\left(2 \mathrm{H}, \mathrm{m}, 4-\mathrm{H}_{2}\right), 1.28(30 \mathrm{H}, \mathrm{m}$, $\left.5-\sim 19-\mathrm{H}_{2}\right), 0.90\left(3 \mathrm{H}, \mathrm{t}, J=6.3 \mathrm{~Hz}, 20-\mathrm{H}_{3}\right)$. FAB-MS $m / z$ $388\left[(\mathrm{M}+\mathrm{H})^{+}\right], 370,328,310,118,57,43$.

18: MP $60 \sim 60.5^{\circ} \mathrm{C}$. $[\alpha]_{\mathrm{D}}^{25}-23.21^{\circ}\left(\right.$ c $\left.1.23, \mathrm{CHCl}_{3}\right)$. IR $v_{\text {max }}\left(\mathrm{CHCl}_{3}\right) \mathrm{cm}^{-1}: 3570(\mathrm{sh}), 3450(\mathrm{sh}), 3390,3000$, 2915, 2835, 1730, 1225. ${ }^{1} \mathrm{H}$ NMR (CD $\mathrm{OD}, 270 \mathrm{MHz}$ ) $\delta: 3.85(1 \mathrm{H}, \mathrm{d}, J=10.6 \mathrm{~Hz}, 21-\mathrm{H}), 3.74(4 \mathrm{H}, \mathrm{m}, 3-\mathrm{H}$ and $\mathrm{OMe}), 3.51(1 \mathrm{H}, \mathrm{d}, J=10.6 \mathrm{~Hz}, 21-\mathrm{H}), 1.56(1 \mathrm{H}, \mathrm{m}, 4-\mathrm{H})$, $1.29\left(31 \mathrm{H}, \mathrm{m}, 4-\mathrm{H}, 5-\sim 19-\mathrm{H}_{2}\right), 0.90(3 \mathrm{H}, \mathrm{t}, J=6.3 \mathrm{~Hz}$, $\left.20-\mathrm{H}_{3}\right)$. FAB-MS $m / z: 388\left[(\mathrm{M}+\mathrm{H})^{+}\right], 370,328,118$, $55,43$.

19: $\mathrm{MP} 69 \sim 71^{\circ} \mathrm{C} .[\alpha]_{\mathrm{D}}^{25}-5.01^{\circ}\left(c 0.621, \mathrm{CHCl}_{3}\right)$. IR $v_{\max }\left(\mathrm{CHCl}_{3}\right) \mathrm{cm}^{-1}: 3605$ (sh), 3500 (sh), 3390, 3000, 2910, 2840, 1735, 1225. ${ }^{1} \mathrm{H}$ NMR (CD $\mathrm{OD}, 270 \mathrm{MHz}$ ) $\delta: 3.88(1 \mathrm{H}, \mathrm{d}, J=10.9 \mathrm{~Hz}, 21-\mathrm{H}), 3.73(3 \mathrm{H}, \mathrm{s}, \mathrm{OMe})$, $3.67(1 \mathrm{H}, \mathrm{dd}, J=10.2$ and $2.0 \mathrm{~Hz}, 3-\mathrm{H}), 3.64(1 \mathrm{H}, \mathrm{d}$, $J=10.9 \mathrm{~Hz}, 21-\mathrm{H}), 1.51\left(2 \mathrm{H}, \mathrm{m}, 4-\mathrm{H}_{2}\right), 1.28(30 \mathrm{H}, \mathrm{m}$, 5- 19- $\left.\mathrm{H}_{2}\right), 0.90\left(3 \mathrm{H}, \mathrm{t}, J=6.3 \mathrm{~Hz}, 20-\mathrm{H}_{3}\right)$. FAB-MS $m / z 388\left[(\mathrm{M}+\mathrm{H})^{+}\right], 370,328,310,118,57,43$.

Methyl 2-Benzamido-3-benzoyloxy-2-benzoyloxymethyleicosanoates $(12(2 S, 3 R), 13(2 S, 3 S), \mathbf{2 0}(2 R, 3 S)$ and $21(2 R, 3 R))$

Benzoic anhydride $(90 \mathrm{mg})$ was added to a solution of $10(12 \mathrm{mg}), 11(3.5 \mathrm{mg}), 18(5.0 \mathrm{mg})$ or $19(5.0 \mathrm{mg})$ and $N, N$-dimethylaminopyridine $(11 \mathrm{mg})$ in $\mathrm{Et}_{3} \mathrm{~N}(1.0 \mathrm{ml})$ and the mixture was stirred at $40^{\circ} \mathrm{C}$ for 2 hours. Ice-water was added and the mixture was extracted with $\mathrm{CHCl}_{3}$. The organic layer was washed and concentrated to give an oily residue. The residue was purified by prepara- tive TLC [solvent, $n$-hexane-EtOAc $(6: 4)]$ to give $\mathbf{1 2}$ (16.8 mg, 77.5\%), $13(3.0 \mathrm{mg}, 47.5 \%), 20(6.3 \mathrm{mg}, 69.8 \%)$ or $21(5.7 \mathrm{mg}, 63.1 \%)$, respectively, as an oil.

12: $[\alpha]_{\mathrm{D}}^{25}+4.83^{\circ}\left(c 0.116, \mathrm{CHCl}_{3}\right) . \mathrm{CD} \lambda_{\mathrm{ext}}(\mathrm{EtOH})$ nm: $241(\Delta \varepsilon=-3.70), 225(3.13)$. IR $v_{\max }\left(\mathrm{CHCl}_{3}\right) \mathrm{cm}^{-1}$ : $3395,3000,2910,2840,1720,1670,1265,710 .{ }^{1} \mathrm{H}$ NMR $\left(\mathrm{CDCl}_{3}, 270 \mathrm{MHz}\right) \delta: 7.99 \sim 7.19(16 \mathrm{H}, \mathrm{m}, \mathrm{NH}$ and aromatic $\mathrm{H}), 5.84(1 \mathrm{H}$, dd, $J=10.6$ and $2.3 \mathrm{~Hz}, 3-\mathrm{H})$, $5.39(1 \mathrm{H}, \mathrm{d}, J=11.7 \mathrm{~Hz}, 21-\mathrm{H}), 5.01(1 \mathrm{H}, \mathrm{d}, J=11.7 \mathrm{~Hz}$, $21-\mathrm{H}), 3.86(3 \mathrm{H}, \mathrm{s}, \mathrm{OMe}), 1.91(1 \mathrm{H}, \mathrm{m}, 4-\mathrm{H}), 1.75(1 \mathrm{H}$, $\mathrm{m}, 4-\mathrm{H}), 1.25\left(30 \mathrm{H}, \mathrm{m}, 5-\sim 19-\mathrm{H}_{2}\right), 0.88(3 \mathrm{H}, \mathrm{t}, J=6.3$ $\left.\mathrm{Hz}, 20-\mathrm{H}_{3}\right)$. FAB-MS $m / z: 700\left[(\mathrm{M}+\mathrm{H})^{+}\right], 578,105$. HRFAB-MS Calcd for $\mathrm{C}_{43} \mathrm{H}_{58} \mathrm{NO}_{7}: 700.4215[(\mathrm{M}+$ $\mathrm{H}^{+}$]. Found: $m / z 700.4219$.

13: $[\alpha]_{\mathrm{D}}^{25}+44.04^{\circ}\left(c 0.045, \mathrm{CHCl}_{3}\right) \cdot \mathrm{CD} \lambda_{\text {ext }}(\mathrm{EtOH})$ nm: $238(\Delta \varepsilon=11.3), 221(-3.65)$. IR $v_{\max }\left(\mathrm{CHCl}_{3}\right) \mathrm{cm}^{-1}$ : $3395,3000,2910,2840,1725,1665,1270,710 .{ }^{1} \mathrm{H}$ NMR $\left(\mathrm{CDCl}_{3}, 270 \mathrm{MHz}\right) \delta: 8.08 \sim 7.39(16 \mathrm{H}, \mathrm{m}, \mathrm{NH}$ and aromatic $\mathrm{H}), 5.93(1 \mathrm{H}$, dd, $J=9.2$ and $4.6 \mathrm{~Hz}, 3-\mathrm{H}), 5.17$ $\left(2 \mathrm{H}, \mathrm{s}, 21-\mathrm{H}_{2}\right), 3.85(3 \mathrm{H}, \mathrm{s}, \mathrm{OMe}), 1.89\left(2 \mathrm{H}, \mathrm{m}, 4-\mathrm{H}_{2}\right)$, $1.25\left(30 \mathrm{H}, \mathrm{m}, 5-\sim 19-\mathrm{H}_{2}\right), 0.88\left(3 \mathrm{H}, \mathrm{t}, J=6.3 \mathrm{~Hz}, 20-\mathrm{H}_{3}\right)$, FAB-MS $m / z: 700\left[(\mathrm{M}+\mathrm{H})^{+}\right], 578,105$. HRFAB-MS Calcd for $\mathrm{C}_{43} \mathrm{H}_{58} \mathrm{NO}_{7}: 700.4215\left[(\mathrm{M}+\mathrm{H})^{+}\right]$. Found: $m / z 700.4224$.

20: $[\alpha]_{\mathrm{D}}^{25}-3.80^{\circ}\left(c 0.111, \mathrm{CHCl}_{3}\right) \cdot \mathrm{CD} \lambda_{\text {ext }}(\mathrm{EtOH})$ $\mathrm{nm}: 241(\Delta \varepsilon=3.83), 225(-3.07)$. IR $v_{\max }\left(\mathrm{CHCl}_{3}\right) \mathrm{cm}^{-1}$ : $3395,3000,2910,2840,1720,1670,1265,710 .{ }^{1} \mathrm{H}$ NMR $\left(\mathrm{CDCl}_{3}, 270 \mathrm{MHz}\right) \delta: 8.01 \sim 7.21(16 \mathrm{H}, \mathrm{m}, \mathrm{NH}$ and aromatic $\mathrm{H}), 5.84(1 \mathrm{H}, \mathrm{dd}, J=10.6$ and $2.3 \mathrm{~Hz}, 3-\mathrm{H})$, $5.39(1 \mathrm{H}, \mathrm{d}, J=11.7 \mathrm{~Hz}, 21-\mathrm{H}), 5.01(1 \mathrm{H}, \mathrm{d}, J=11.7 \mathrm{~Hz}$, 21-H), $3.86(3 \mathrm{H}, \mathrm{s}, \mathrm{OMe}), 1.91(1 \mathrm{H}, \mathrm{m}, 4-\mathrm{H}), 1.75(1 \mathrm{H}$, $\mathrm{m}, 4-\mathrm{H}), 1.25\left(30 \mathrm{H}, \mathrm{m}, 5-\sim 19-\mathrm{H}_{2}\right), 0.88(3 \mathrm{H}, \mathrm{t}, J=6.3$ $\left.\mathrm{Hz}, 20-\mathrm{H}_{3}\right)$. FAB-MS $m / z: 700\left[(\mathrm{M}+\mathrm{H})^{+}\right], 578,105$. HRFAB-MS Calcd for $\mathrm{C}_{43} \mathrm{H}_{58} \mathrm{NO}_{7}: 700.4215[(\mathrm{M}+$ $\mathrm{H}^{+}$]. Found: $m / z 700.4203$.

21: $\left.[\alpha]_{\mathrm{D}}^{25}-34.36^{\circ}(c) 0.103, \mathrm{CHCl}_{3}\right) . \quad \mathrm{CD} \lambda_{\mathrm{ext}}$ $(\mathrm{EtOH}) \mathrm{nm}: 238(\Delta \varepsilon=-11.0), 222(-3.01) . \quad$ IR $v_{\max }$ $\left(\mathrm{CHCl}_{3}\right) \mathrm{cm}^{-1}: 3395,3000,2910,2840,1725,1665,1265$, 710. ${ }^{1} \mathrm{H} \mathrm{NMR}\left(\mathrm{CDCl}_{3}, 270 \mathrm{MHz}\right) \delta: 8.08 \sim 7.38(16 \mathrm{H}$, $\mathrm{m}, \mathrm{NH}$ and aromatic $\mathrm{H}), 5.93(1 \mathrm{H}, \mathrm{dd}, J=9.2$ and $4.6 \mathrm{~Hz}$, $3-\mathrm{H}), 5.17\left(2 \mathrm{H}, \mathrm{s}, 21-\mathrm{H}_{2}\right), 3.85(3 \mathrm{H}, \mathrm{s}, \mathrm{OMe}), 1.88(2 \mathrm{H}$, $\left.\mathrm{m}, 4-\mathrm{H}_{2}\right), 1.25\left(30 \mathrm{H}, \mathrm{m}, 5-\sim 19-\mathrm{H}_{2}\right), 0.88(3 \mathrm{H}, \mathrm{t}, J=$ $\left.6.3 \mathrm{~Hz}, 20-\mathrm{H}_{3}\right)$, FAB-MS $m / z: 700\left[(\mathrm{M}+\mathrm{H})^{+}\right], 578$, 105. HRFAB-MS Calcd for $\mathrm{C}_{43} \mathrm{H}_{58} \mathrm{NO}_{7}: 700.4215$ $\left[(\mathrm{M}+\mathrm{H})^{+}\right]$. Found: $m / z 700.4216$.

$N, O, O^{\prime}$-Tribenzoyl Methyl Esters of 2, 3, 4 and $\mathbf{5}(\mathbf{2 2}$, 23, 24 and 25)

Excess ethereal diazomethane was added to a solution of $2(29.4 \mathrm{mg}), 3(10 \mathrm{mg}), \mathbf{4}(22.4 \mathrm{mg})$ or $5(10 \mathrm{mg})$ in $\mathrm{MeOH}(50 \mathrm{ml})$ at $0^{\circ} \mathrm{C}$ and the mixture was kept standing at room temperature for 15 minutes. The solvent was evaporated off and the residue was purified by preparative TLC [solvent, $\mathrm{CHCl}_{3}-\mathrm{MeOH}(9: 1)$ ] to give the corresponding methyl ester $(27.4 \mathrm{mg}, 3.8 \mathrm{mg}, 19.1 \mathrm{mg}$ or $4.6 \mathrm{mg}$ ) of $2,3,4$ or 5 , respectively, as an oil.

By a procedure similar to that used for the preparation of 12, 13, 20 and 21, 22 (14.4 mg), 23 (9.4 mg), 24 (3.2 mg) 
and $25(3.7 \mathrm{mg})$ were obtained as oils in $72.7 \%, 69.8 \%$, $43.4 \%$ and $63.1 \%$ yields from the above methyl esters $(10 \mathrm{mg}, 3.8 \mathrm{mg}, 10 \mathrm{mg}$ and $4.6 \mathrm{mg}$ ) of $2,3,4$ and 5 , respectively.

22: $[\alpha]_{\mathrm{D}}^{25}+38.60^{\circ}\left(c 0.251, \mathrm{CHCl}_{3}\right) . \mathrm{CD} \lambda_{\mathrm{ext}}(\mathrm{EtOH})$ $\mathrm{nm}: 240(\Delta \varepsilon=10.5), 223(-3.69)$. IR $v_{\max }\left(\mathrm{CHCl}_{3}\right) \mathrm{cm}^{-1}$ : $3395,3000,2910,2840,1725,1665,1265,970,710 .{ }^{1} \mathrm{H}$ NMR $\left(\mathrm{CDCl}_{3}, 270 \mathrm{MHz}\right) \delta: 8.09 \sim 7.37(16 \mathrm{H}, \mathrm{m}, \mathrm{NH}$ and aromatic $\mathrm{H}), 5.91(1 \mathrm{H}, \mathrm{dd}, J=8.9$ and $4.0 \mathrm{~Hz}, 3-\mathrm{H})$, $5.31(2 \mathrm{H}, \mathrm{m}, 6-$ and $7-\mathrm{H}) 5.17(1 \mathrm{H}, \mathrm{d}, J=11.7 \mathrm{~Hz}, 21-\mathrm{H})$, $5.15(1 \mathrm{H}, \mathrm{d}, J=11.7 \mathrm{~Hz}, 21-\mathrm{H}), 3.85(3 \mathrm{H}, \mathrm{s}, \mathrm{OMe}), 2.37$ $\left(2 \mathrm{H}, \mathrm{t}, J=7.3 \mathrm{~Hz}, 13-\right.$ or $\left.15-\mathrm{H}_{2}\right), 2.36(2 \mathrm{H}, \mathrm{t}, J=7.3 \mathrm{~Hz}$, 13- or $\left.15-\mathrm{H}_{2}\right), 2.12(1 \mathrm{H}, \mathrm{m}, 5-\mathrm{H}), 1.98(3 \mathrm{H}, \mathrm{m}, 5-\mathrm{H}$ and $\left.8-\mathrm{H}_{2}\right), 1.81\left(2 \mathrm{H}, \mathrm{m}, 4-\mathrm{H}_{2}\right), 1.53\left(4 \mathrm{H}, \mathrm{m}, 12-\right.$ and $\left.16-\mathrm{H}_{2}\right)$, $1.27\left(12 \mathrm{H}, \mathrm{m}, 9-\sim 11-\right.$ and $\left.17-\sim 19-\mathrm{H}_{2}\right), 0.88(3 \mathrm{H}, \mathrm{t}$, $\left.J=6.3 \mathrm{~Hz}, 20-\mathrm{H}_{3}\right)$. FAB-MS $m / z: 712\left[(\mathrm{M}+\mathrm{H})^{+}\right], 590$, 105. HRFAB-MS Calcd for $\mathrm{C}_{43} \mathrm{H}_{54} \mathrm{NO}_{8}$ : 712.3851 $\left[(\mathrm{M}+\mathrm{H})^{+}\right]$. Found: $m / z 712.3860$.

23: $[\alpha]_{\mathrm{D}}^{25}+37.61^{\circ}\left(c 0.031, \mathrm{CHCl}_{3}\right) \cdot \mathrm{CD} \lambda_{\text {ext }}(\mathrm{EtOH})$ $\mathrm{nm}: 239(\Delta \varepsilon=10.5), 221(-3.41) . \mathrm{IR} v_{\max }\left(\mathrm{CHCl}_{3}\right) \mathrm{cm}^{-1}$ : $3400,3000,2910,2845,1725,1665,1265,710 .{ }^{1} \mathrm{H}$ NMR $\left(\mathrm{CDCl}_{3}, 270 \mathrm{MHz}\right) \delta: 8.08 \sim 7.36(16 \mathrm{H}, \mathrm{m}, \mathrm{NH}$ and aromatic $\mathrm{H}), 5.94(1 \mathrm{H}, \mathrm{dd}, J=9.6$ and $4.3 \mathrm{~Hz}, 3-\mathrm{H}), 5.18$ $(1 \mathrm{H}, \mathrm{d}, J=11.9 \mathrm{~Hz}, 21-\mathrm{H}), 5.16(1 \mathrm{H}, \mathrm{d}, J=11.9 \mathrm{~Hz}$, $21-\mathrm{H}), 3.85(3 \mathrm{H}, \mathrm{s}, \mathrm{OMe}), 2.37(2 \mathrm{H}, \mathrm{t}, J=7.3 \mathrm{~Hz}, 13-$ or $\left.15-\mathrm{H}_{2}\right), 2.36\left(2 \mathrm{H}, \mathrm{t}, J=7.3 \mathrm{~Hz}, 13-\right.$ or $\left.15-\mathrm{H}_{2}\right), 1.88(2 \mathrm{H}$, $\left.\mathrm{m}, 4-\mathrm{H}_{2}\right), 1.53\left(4 \mathrm{H}, \mathrm{m}, 12-\right.$ and $\left.16-\mathrm{H}_{2}\right), 1.26(20 \mathrm{H}, \mathrm{m}$, 5- 11- and 17- 19- $\left.\mathrm{H}_{2}\right), 0.88\left(3 \mathrm{H}, \mathrm{t}, J=6.3 \mathrm{~Hz}, 20-\mathrm{H}_{3}\right)$. FAB-MS $m / z: 714\left[(\mathrm{M}+\mathrm{H})^{+}\right]$. HRFAB-MS Calcd for $\mathrm{C}_{43} \mathrm{H}_{56} \mathrm{NO}_{8}: 714.4008\left[(\mathrm{M}+\mathrm{H})^{+}\right]$. Found: $m / z$ 714.4020 .

24: $[\alpha]_{\mathrm{D}}^{25}+6.02^{\circ}\left(c 0.138, \mathrm{CHCl}_{3}\right) . \mathrm{CD} \lambda_{\text {ext }}(\mathrm{EtOH})$ $\mathrm{nm}: 241(\Delta \varepsilon=-3.46), 226(3.33) . \mathrm{IR} v_{\max }\left(\mathrm{CHCl}_{3}\right) \mathrm{cm}^{-1}$ : $3390,3000,2910,2840,1720,1670,1265,965,710 .{ }^{1} \mathbf{H}$ NMR $\left(\mathrm{CDCl}_{3}, 270 \mathrm{MHz}\right) \delta: 7.99 \sim 7.17(16 \mathrm{H}, \mathrm{m}, \mathrm{NH}$ and aromatic $\mathrm{H}), 5.81(1 \mathrm{H}, \mathrm{dd}, J=10.2$ and $2.3 \mathrm{~Hz}, 3-\mathrm{H})$, $5.38(1 \mathrm{H}, \mathrm{d}, J=11.5 \mathrm{~Hz}, 21-\mathrm{H}), 5.33(2 \mathrm{H}, \mathrm{m}, 6-, 7-\mathrm{H})$, $5.01(1 \mathrm{H}, \mathrm{d}, J=11.5 \mathrm{~Hz}, 21-\mathrm{H}), 3.86(3 \mathrm{H}, \mathrm{s}, \mathrm{OMe}), 2.37$ $\left(2 \mathrm{H}, \mathrm{t}, J=7.3 \mathrm{~Hz}, 13-\right.$ or $\left.15-\mathrm{H}_{2}\right), 2.35(2 \mathrm{H}, \mathrm{t}, J=7.3 \mathrm{~Hz}$, 13- or $\left.15-\mathrm{H}_{2}\right), 2.07\left(3 \mathrm{H}, \mathrm{m}, 5-\mathrm{H}\right.$ and $\left.8-\mathrm{H}_{2}\right), 1.87(3 \mathrm{H}, \mathrm{m}$, $4-\mathrm{H}_{2}$ and $\left.5-\mathrm{H}\right), 1.53\left(4 \mathrm{H}, \mathrm{m}, 12-\right.$ and $\left.16-\mathrm{H}_{2}\right), 1.26(12 \mathrm{H}$, $\mathrm{m}, 9-\sim 11-$ and $\left.17-\sim 19-\mathrm{H}_{2}\right), 0.87(3 \mathrm{H}, \mathrm{t}, J=6.6 \mathrm{~Hz}, 20-$ $\mathrm{H}_{3}$ ). FAB-MS $m / z: 712\left[(\mathrm{M}+\mathbf{H})^{+}\right], 590,105$. HRFABMS Calcd for $\mathrm{C}_{43} \mathrm{H}_{54} \mathrm{NO}_{8}$ : 712.3851 $\left[(\mathrm{M}+\mathrm{H})^{+}\right]$. Found: $m / z 712.3839$.

25: $[\alpha]_{\mathrm{D}}^{25}+5.78^{\circ}\left(c 0.054, \mathrm{CHCl}_{3}\right) \cdot \mathrm{CD} \hat{\lambda}_{\text {ext }}(\mathrm{EtOH})$ $\mathrm{nm}: 239(\Delta \varepsilon=-3.25), 226(2.84)$. IR $v_{\max }\left(\mathrm{CHCl}_{3}\right) \mathrm{cm}^{-1}$ : $3390,3000,2910,2840,1720,1670,1265,710 .{ }^{1} \mathrm{H}$ NMR $\left(\mathrm{CDCl}_{3}, 270 \mathrm{MHz}\right) \delta: 7.99 \sim 7.17(16 \mathrm{H}, \mathrm{m}, \mathrm{NH}$ and aromatic $\mathrm{H}), 5.85(1 \mathrm{H}$, dd, $J=10.9$ and $2.6 \mathrm{~Hz}, 3-\mathrm{H})$, $5.39(1 \mathrm{H}, \mathrm{d}, J=11.5 \mathrm{~Hz}, 21-\mathrm{H}), 5.01(1 \mathrm{H}, \mathrm{d}, J=11.5 \mathrm{~Hz}$, $21-\mathrm{H}), 3.87(3 \mathrm{H}, \mathrm{s}, \mathrm{OMe}), 2.37(2 \mathrm{H}, \mathrm{t}, J=7.6 \mathrm{~Hz}, 13$ - or $\left.15-\mathrm{H}_{2}\right), 2.35\left(2 \mathrm{H}, \mathrm{t}, J=7.3 \mathrm{~Hz}, 13-\right.$ or $\left.15-\mathrm{H}_{2}\right), 1.90(1 \mathrm{H}$, $\mathrm{m}, 4-\mathrm{H}), 1.74(1 \mathrm{H}, \mathrm{m}, 4-\mathrm{H}), 1.55\left(4 \mathrm{H}, \mathrm{m}, 12-\right.$ and $\left.16-\mathrm{H}_{2}\right)$, $1.20\left(12 \mathrm{H}, \mathrm{m}, 5-\sim 11-\right.$ and $\left.17-\sim 19-\mathrm{H}_{2}\right), 0.87(3 \mathrm{H}, \mathrm{t}$, $\left.J=6.6 \mathrm{~Hz}, 20-\mathrm{H}_{3}\right)$. FAB-MS $m / z: 714\left[(\mathrm{M}+\mathrm{H})^{+}\right]$. HRFAB-MS Calcd for $\mathrm{C}_{43} \mathrm{H}_{56} \mathrm{NO}_{8}: 714.4008[(\mathrm{M}+$ $\mathrm{H})^{+}$]. Found: $m / z 714.4001$.
(R)-MTPA Ester of 8 (26)

$N, N$-Dimethylaminopyridine $(7.3 \mathrm{mg}), \mathrm{Et}_{3} \mathrm{~N}(3.1 \mu \mathrm{l})$ and $(S)-(+)$-MTPA chloride $(5.6 \mu l)$ were added to a solution of $8(7.3 \mathrm{mg})$ in dry $\mathrm{CH}_{2} \mathrm{Cl}_{2}(0.5 \mathrm{ml})$, and the mixture was stirred at room temperature for 4 hours. 3-(Dimethylamino)propylamine $(3.8 \mu \mathrm{l})$ was added to the mixture, then the solvent was evaporated off. The residue was purified by preparative TLC [solvent, $n$-hexane-EtOAc $(8: 2)]$ to give $\mathbf{2 6}$ as an oil $(7.1 \mathrm{mg}$, $66.0 \%) . I R v_{\max }\left(\mathrm{CHCl}_{3}\right) \mathrm{cm}^{-1}: 2910,2840,1755,1675$, $1220,710 .{ }^{1} \mathrm{H} \mathrm{NMR}\left(\mathrm{CDCl}_{3}, 270 \mathrm{MHz}\right) \delta: 8.51,8.06$ $(1 \mathrm{H}, 2 \mathrm{~s}, \mathrm{CHO}), 7.53 \sim 7.51(2 \mathrm{H}, \mathrm{m}$, aromatic $\mathrm{H})$, $7.43 \sim 7.41(3 \mathrm{H}, \mathrm{m}$, aromatic $\mathrm{H}), 6.37,5.67(1 \mathrm{H}, \mathrm{m}$ and $\mathrm{dd}, J=10.0$ and $\left.2.3 \mathrm{~Hz}, 1^{\prime}-\mathrm{H}\right), 5.33,4.34(1 \mathrm{H}, 2 \mathrm{~s}, 2-\mathrm{H})$, $4.44(1 \mathrm{H}, \mathrm{d}, J=9.9 \mathrm{~Hz}, 5-\mathrm{H}), 4.02(1 \mathrm{H}, \mathrm{d}, J=9.9 \mathrm{~Hz}$, $5-\mathrm{H}), 3.75,3.72(3 \mathrm{H}, 2 \mathrm{~s}, \mathrm{COOMe}), 3.48,3.41(3 \mathrm{H}, 2 \mathrm{q}$, $J=1.3 \mathrm{~Hz}, \mathrm{OMe}), 1.59\left(1 \mathrm{H}, \mathrm{m}, 2^{\prime}-\mathrm{H}\right), 1.40\left(1 \mathrm{H}, \mathrm{m}, 2^{\prime}-\mathrm{H}\right)$, $1.26\left(30 \mathrm{H}, \mathrm{m}, 3^{\prime}-\sim 17^{\prime}-\mathrm{H}_{2}\right), 0.93,0.89(9 \mathrm{H}, 2 \mathrm{~s}, t-\mathrm{Bu})$, $0.88\left(3 \mathrm{H}, \mathrm{t}, J=6.9 \mathrm{~Hz}, 18^{\prime}-\mathrm{H}_{3}\right)$. FAB-MS $m / z: 700$ $\left[(\mathrm{M}+\mathrm{H})^{+}\right], 670,614,380,189$. HRFAB-MS Calcd for $\mathrm{C}_{38} \mathrm{H}_{61} \mathrm{NO}_{7} \mathrm{~F}_{3}: 700.4402\left[(\mathrm{M}+\mathrm{H})^{+}\right]$. Found: $m / z$ 700.4396 .

\section{$(S)$-MTPA Ester of 8 (27)}

By a procedure similar to that used for the preparation of 26, $27(6.1 \mathrm{mg})$ was obtained as an oil in $58.1 \%$ yield from $8(7.3 \mathrm{mg})$ and $(R)-(-)$-MTPA chloride $(5.6 \mu 1)$ IR $v_{\max }\left(\mathrm{CHCl}_{3}\right) \mathrm{cm}^{-1}: 2910,2840,1755,1675,1230$, 710. ${ }^{1} \mathrm{H} \mathrm{NMR}\left(\mathrm{CDCl}_{3}, 270 \mathrm{MHz}\right) \delta: 8.50,7.90(1 \mathrm{H}, 2 \mathrm{~s}$, $\mathrm{CHO}), 7.53 \sim 7.52(2 \mathrm{H}, \mathrm{m}$, aromatic $\mathrm{H}), 7.43 \sim 7.39$ $(3 \mathrm{H}, \mathrm{m}$, aromatic $\mathrm{H}), 6.37,5.68(1 \mathrm{H}, \mathrm{m}$ and $\mathrm{dd}, J=9.9$ and $\left.2.3 \mathrm{~Hz}, 1^{\prime}-\mathrm{H}\right), 5.35,4.26(1 \mathrm{H}, 2 \mathrm{~s}, 2-\mathrm{H}), 4.42,4.40$ $(1 \mathrm{H}, 2 \mathrm{~d}, J=9.9 \mathrm{~Hz}, 5-\mathrm{H}), 4.08,4.03(1 \mathrm{H}, 2 \mathrm{~d}, J=9.9 \mathrm{~Hz}$, $5-\mathrm{H}), 3.75,3.58(3 \mathrm{H}, 2 \mathrm{~s}, \mathrm{COOMe}), 3.62,3.52(3 \mathrm{H}, 2 \mathrm{q}$, $J=1.3 \mathrm{~Hz}, \mathrm{OMe}), 1.65\left(1 \mathrm{H}, \mathrm{m}, 2^{\prime}-\mathrm{H}\right), 1.47\left(1 \mathrm{H}, \mathrm{m}, 2^{\prime}-\mathrm{H}\right)$, $1.26\left(30 \mathrm{H}, \mathrm{m}, 3^{\prime}-\sim 17^{\prime}-\mathrm{H}_{2}\right), 0.92,0.84(9 \mathrm{H}, 2 \mathrm{~s}, t-\mathrm{Bu})$, $0.88\left(3 \mathrm{H}, \mathrm{t}, J=6.9 \mathrm{~Hz}, 18^{\prime}-\mathrm{H}_{3}\right)$. FAB-MS $m / z: 700$ $\left[(\mathrm{M}+\mathrm{H})^{+}\right], 670,614,380,189$. HRFAB-MS Calcd for $\mathrm{C}_{38} \mathrm{H}_{61} \mathrm{NO}_{7} \mathrm{~F}_{3}: 700.4402\left[(\mathrm{M}+\mathrm{H})^{+}\right]$. Found: $m / z$ 700.4415 .

(R)-MTPA Ester of N,21-O-Diacetyl Mycestericin E Methyl Ester (28)

Pyridine $(1.0 \mathrm{ml})$ and acetic anhydride $(1.0 \mathrm{ml})$ were added to a solution of methyl ester of $4(20.0 \mathrm{mg})$ in $\mathrm{CH}_{2} \mathrm{Cl}_{2}(1.5 \mathrm{ml})$ at $0^{\circ} \mathrm{C}$, and the mixture was kept standing at $0^{\circ} \mathrm{C}$ for 3 hours. Ice-water was added and the whole was extracted with $\mathrm{CHCl}_{3}$. The organic solution was washed and concentrated to give an oily residue. The residue was purified by preparative TLC [solvent, $\left.\mathrm{CHCl}_{3}-\mathrm{MeOH}(97: 3)\right]$ to give the $N, 21-O$-diacetyl compound as an oil ( $9.4 \mathrm{mg}, 38.8 \%$ ).

By a procedure similar to that used for the preparation of $26,28(3.1 \mathrm{mg})$ was obtained as an oil in $42.8 \%$ yield from the diacetyl compound $(5.0 \mathrm{mg}) . \mathrm{IR} v_{\max }\left(\mathrm{CHCl}_{3}\right)$ $\mathrm{cm}^{-1}: 3400,3000,2910,2845,1745,1715,1685,1270$ (sh), 1220, 715. ${ }^{1} \mathrm{H}$ NMR $\left(\mathrm{CDCl}_{3}, 270 \mathrm{MHz}\right) \delta: 7.58 \sim$ $7.57(2 \mathrm{H}, \mathrm{m}$, aromatic $\mathrm{H}), 7.44 \sim 7.41(3 \mathrm{H}, \mathrm{m}$, aromatic 
H), $6.19(1 \mathrm{H}$, br s, NH), $5.74(1 \mathrm{H}, \mathrm{dd}, J=9.7$ and $2.5 \mathrm{~Hz}$, $3-\mathrm{H}), 5.36(1 \mathrm{H}, \mathrm{dt}, J=15.3$ and $6.6 \mathrm{~Hz}, 7-\mathrm{H}), 5.26(1 \mathrm{H}$, $\mathrm{dt}, J=15.3$ and $6.4 \mathrm{~Hz}, 6-\mathrm{H}), 4.93(1 \mathrm{H}, \mathrm{d}, J=11.3 \mathrm{~Hz}$, $21-\mathrm{H}), 4.48(1 \mathrm{H}, \mathrm{d}, J=11.3 \mathrm{~Hz}, 21-\mathrm{H}), 3.72(3 \mathrm{H}, \mathrm{s}$, COOMe), $3.51(3 \mathrm{H}, \mathrm{s}, \mathrm{OMe}), 2.37(4 \mathrm{H}, \mathrm{t}, J=7.4 \mathrm{~Hz}$, 13- and 15- $\left.\mathrm{H}_{2}\right), 1.99(3 \mathrm{H}, \mathrm{s}, \mathrm{Ac}), 1.96(3 \mathrm{H}, \mathrm{s}, \mathrm{Ac}), 1.94$ $\left(2 \mathrm{H}, \mathrm{m}, 8-\mathrm{H}_{2}\right), 1.93\left(2 \mathrm{H}, \mathrm{m}, 5-\mathrm{H}_{2}\right), 1.78(1 \mathrm{H}, \mathrm{m}, 4-\mathrm{H})$, $1.65(1 \mathrm{H}, \mathrm{m}, 4-\mathrm{H}), 1.56(4 \mathrm{H}$, qui, $J=7.2 \mathrm{~Hz}, 12-$ and $\left.16-\mathrm{H}_{2}\right), 1.32\left(2 \mathrm{H}, \mathrm{m}, 9-\mathrm{H}_{2}\right), 1.28(10 \mathrm{H}, \mathrm{m}, 10-, 11-$ and $\left.17-\sim 19-\mathrm{H}_{2}\right), 0.88\left(3 \mathrm{H}, \mathrm{t}, J=7.0 \mathrm{~Hz}, 20-\mathrm{H}_{2}\right)$. FAB-MS $m / z: 700\left[(\mathrm{M}+\mathrm{H})^{+}\right], 640,466,189,43$. HRFAB-MS Calcd for $\mathrm{C}_{36} \mathrm{H}_{53} \mathrm{NO}_{9} \mathrm{~F}_{3}: 700.3674\left[(\mathrm{M}+\mathrm{H})^{+}\right]$. Found: $m / z 700.3663$.

(S)-MTPA Ester of $N, 21-O$-Diacetyl Mycestericin E Methyl Ester (29)

By a procedure similar to that used for the preparation of $28,29(3.9 \mathrm{mg})$ was obtained as an oil in $58.1 \%$ yield from $N, 21-O$-diacetyl mycestericin $\mathrm{E}$ methyl ester $(5.0 \mathrm{mg})$ and $(R)-(-)$-MTPA chloride $(5.6 \mu \mathrm{l})$. IR $v_{\max }$ $\left(\mathrm{CHCl}_{3}\right) \mathrm{cm}^{-1}: 3400,3000,2910,2850,1745,1710$, $1685,1250(\mathrm{sh}), 1220,720 .{ }^{1} \mathrm{H}$ NMR $\left(\mathrm{CDCl}_{3}, 270 \mathrm{MHz}\right)$ $\delta: 7.57 \sim 7.56(2 \mathrm{H}, \mathrm{m}$, aromatic $\mathrm{H}), 7.43 \sim 7.41(3 \mathrm{H}, \mathrm{m}$; aromatic $\mathrm{H}), 6.09(1 \mathrm{H}$, br s, $\mathrm{NH}), 5.73(1 \mathrm{H}, \mathrm{dd}, J=9.4$ and $2.6 \mathrm{~Hz}, 3-\mathrm{H}), 5.38(1 \mathrm{H}, \mathrm{dt}, J=15.3$ and $6.5 \mathrm{~Hz}, 7-\mathrm{H})$, $5.29(1 \mathrm{H}, \mathrm{dt}, J=15.3$ and $6.6 \mathrm{~Hz}, 6-\mathrm{H}), 4.88(1 \mathrm{H}, \mathrm{d}$, $J=11.4 \mathrm{~Hz}, 21-\mathrm{H}), 4.45(1 \mathrm{H}, \mathrm{d}, J=11.4 \mathrm{~Hz}, 21-\mathrm{H}), 3.72$ ( $3 \mathrm{H}, \mathrm{s}, \mathrm{COOMe}), 3.55(3 \mathrm{H}, \mathrm{s}, \mathrm{OMe}), 2.37 \cdot(4 \mathrm{H}, \mathrm{t}, J=$ 7.4Hz, 13- and 15- $\left.\mathrm{H}_{2}\right), 1.99(1 \mathrm{H}, \mathrm{m}, 5-\mathrm{H}), 1.96(3 \mathrm{H}, \mathrm{s}$, Ac), $1.95\left(2 \mathrm{H}, \mathrm{m}, 8-\mathrm{H}_{2}\right), 1.94(1 \mathrm{H}, \mathrm{m}, 5-\mathrm{H}), 1.92(3 \mathrm{H}, \mathrm{s}$, Ac), $1.82(1 \mathrm{H}, \mathrm{m}, 4-\mathrm{H}), 1.71(1 \mathrm{H}, \mathrm{m}, 4-\mathrm{H}), 1.56(4 \mathrm{H}$, qui, $J=7.3 \mathrm{~Hz}, 12-$ and $\left.16-\mathrm{H}_{2}\right), 1.33\left(2 \mathrm{H}, \mathrm{m}, 9-\mathrm{H}_{2}\right), 1.28$ $\left(10 \mathrm{H}, \mathrm{m}, 10-, 11-\right.$ and $\left.17-\sim 19-\mathrm{H}_{2}\right), 0.88(3 \mathrm{H}, \mathrm{t}, J=$ $\left.6.8 \mathrm{~Hz}, 20-\mathrm{H}_{3}\right)$. FAB-MS $m / z: 700\left[(\mathrm{M}+\mathrm{H})^{+}\right], 640,466$, 189, 43. HRFAB-MS Calcd for $\mathrm{C}_{36} \mathrm{H}_{53} \mathrm{NO}_{9} \mathrm{~F}_{3}: 700.3674$ $\left[(\mathrm{M}+\mathrm{H})^{+}\right]$. Found: $m / z 700.3681$.

\section{Acknowledgments}

This study was supported in part by a Grant-in-Aid for Scientific Research (No. 07558090) from the Ministry of Education, Science and Culture, Japan.

\section{References}

1) Kanai, M.; A. Iida, Y. Nagaoka, S. Wada \& T. Fujita: Fungal metabolites. Part 21. Characteristics of low energy collision induced dissociation of $(\mathrm{M}+2 \mathrm{H})^{2+}$, $(\mathrm{M}+\mathrm{H}+\mathrm{Na})^{2+}$ and $(\mathrm{M}+2 \mathrm{Na})^{2+}$ of peptaibols using electrospray ionization mass spectrometry. J. Mass
Spectrometry 31: 177 183, 1966

2) Fujita, T.; K. Inoue, S. Yamamoto, T. Ikumoto, S. Sasaki, R. TOYama, K. Chiba, Y. Hoshino \& $\mathrm{T}$. Oкumoto: Fungal metabolites. Part 11. A potent immunosuppressive activity found in Isaria sinclairii metabolite. J. Antibiotics 47: 208 215, 1994

3) Kluepfel, D.; J. Bagli, H. Baker, M.-P. Charest, A. Kudelski, S. N. Segal \& C. Vezina: Myriocin, a new antifungal antibiotic from Myriococcum albomycès. J. Antibiotics 25: $109 \sim 115,1972$

4) BaGLI, J. F.; D. Kluepfel \& M. St-JACQUes: Elucidation of structure and stereochemistry of myriocin. A novel antifungal antibiotic. J. Org. Chem. 38: $1253 \sim 1260$, 1973

5) Aragozzini, F.; P. L. Manachini, R. Craveri, B. Rindone \& C. SCOLASTiCo: Isolation and structure determination of a new antifungal $\alpha$-hydroxymethyl $\alpha$ amino acid. Tetrahedron 28: $5493 \sim 5498,1972$

6) Sasaki, S.; R. Hashimoto, M. KiUchi, K. Inoue, T. Ikumoto, R. Hirose, K. Chiba, Y. Hoshino, T. Окимото \& T. Fujta: Fungal metabolite. Part 14. Novel potent immunosuppressants, mycestericins, produced by Mycelia sterilia. J. Antibiotics 47: 420 433, 1994

7) Fujita, T.; N. Hamamichi, T. Matsuzaki, Y. Kitao, M. Kivchi, M. Node \& R. Hirose: Determination of the absolute configurations and total synthesis of new immunosuppressants, mycestericins $\mathrm{E}$ and G. Tetrahedron Lett. 36: 8599 8602, 1995

8) SeEbach, D. \& J. D. AEBI: $\alpha$-Alkylation of serine with self-reproduction of the center of chirality. Tetrahedron Lett. 25: 2545 2548, 1984

9) Singh, N.P.; A. Giannis, E. Henk, T. Kolter, K. SANDHOFF \& R. R. SchmidT: Synthesis of 2-carboxysubstituted sphingosine derivatives. J. Carbohydrate Chemistry. 9: $543 \sim 559,1990$

10) Kusumi, T.; Y. Fujta, I. Ohtani \& H. Kakisawa: Anomaly in the modified Mosher's method: absolute configurations of some marine cembranolides. Tetrahedron Lett. 32: 2923 2926, 1991

11) Fujita, T.; M. Yoneta, R. Hirose, S. Sasaki, K. Inoue, M. Kiuchi, S. Hirase, K. Adachi, M. Arita \& K. Chiba: Simple compounds, 2-alkyl-2-amino-1,3-propanediols have potent immunosuppressive activity. BioMed. Chem. Lett. 5: $847 \sim 852,1995$

12) Adachi, K.; T. Kohara, N. NaKao, M. Arita, K. Chiba, T. Mishina, S. Sasaki \& T. Fujita: Design, synthesis, and structure-activity relationships of 2-substituted-2amino-1,3-propanediols: discovery of a novel immunosuppressant, FTY720. BioMed. Chem. Lett. 5: 853 856, 1995 